\title{
Las escuelas al aire libre como contexto para el aprendizaje de las ciencias en infantil. El caso de la Scuola nel BoscoVilla Ghigi
}

\author{
Esther García-González \\ Departamento de Didáctica, Facultad de Ciencias de la Educación. Universidad de Cádiæ, España. \\ esther.garcia@uca.es \\ ORCID: bttps:/ / orcid.org/0000-0003-0995-9798 \\ Michela Schenetti \\ Dipartimento di Scienze Dell'Educaz̧ione "Giovanni Maria Bertin". Università di Bologna, Italia. \\ michela.schenetti@unibo.it \\ ORCID: https:/ / orcid.org/0000-0003-4958-4564
}

[Recibido: 21 Septiembre 2018. Revisado: 19 Diciembre 2018. Aceptado: 19 Marzo 2019]

Resumen: Este trabajo quiere dar a conocer la potencialidad de las escuelas en el bosque para el aprendizaje de las ciencias en las primeras etapas de escolarización. Se presentan los resultados de una investigación desarrollada en la Scuola nel BoscoVilla Ghigi situada en Bolonia (Italia), que cuenta con años de experiencia en escuelas al aire libre. De estos se desprende que este espacio favorece el aprendizaje de las ciencias, pues se identificaron numerosas situaciones donde los niños desplegaron habilidades científicas para solucionar distintas problemáticas. En ellas había implicados una alta diversidad de conocimientos, principalmente de naturaleza procedimental, pero también conceptuales y actitudinales. No obstante, se detectó la necesidad de incluir más espacios para la reflexión que ayuden a asentar y cerrar los aprendizajes.

Palabras clave: aprendizaje de las ciencias, competencia científica, enseñanza al aire libre

Open air schools a context for learning science at the infant stage. The case of the Scuola nel boscoVilla Ghigi

Abstract: This work aims to inform about the potential of forest schools for learning science in the early stages of schooling. We hereby present the results of a study carried out in the Scuola nel Bosco Villa Ghigi in Bologna (Italy) which has been working in this field for years. The results show that this space favors the learning of science. We identified numerous situations where children deployed scientific skills skills to solutions to different problems. They had involved a high diversity of knowledge, mostly of a procedural nature, but also conceptual and attitudinal. However, the need to include more space for reflection to help settle and close the learning process was detected.

Keywords: science learning, scientific competence, open air school education

Para citar este artículo: García-González, E. y Schenetti, M., (2019) Las escuelas al aire libre como contexto para el aprendizaje de las ciencias en infantil. El caso de la Scuola nel BoscoVilla Ghigi. Revista Eureka sobre Enseñanza y Divulgación de las Ciencias 16(2), 2204. doi: 10.25267/Rev_Eureka_ensen_divulg_cienc.2019.v16.i2.2204

\section{Introducción}

Las escuelas en el bosque son una alternativa a los centros de educación infantil tradicionales. Estas escuelas surgieron el siglo pasado en los países escandinavos, extendiéndose posteriormente hacia el sur de Europa (Schenetti, Salvaterra y Rossini 2015). Son numerosas en Italia y en España están en crecimiento. En nuestro país existen experiencias estables y alguna de ellas homologada para la etapa señalada como el caso de Bosquescuela, en Cerceda (Madrid). También existen otras que combinan la escuela tradicional con la escuela en el 
bosque como el proyecto piloto Baso Eskola (Sanz Alonso, Sistiaga, Urkidi, Andoño y Orbegozo 2018).

Estas escuelas aportan abundantes beneficios y de diversa naturaleza. El contacto con la naturaleza es primordial para fortalecer los lazos de los niños con su entorno, de suyo, la primera infancia se considera una etapa de andamiaje para desarrollar un estilo de vida sostenible (Freire, 2011).

Alrededor de estas experiencias educativas son múltiples los temas sobre los cuales se ha puesto el foco de investigación, la salud de los niños (Taylor y Kuo, 2010), el impacto en su desarrollo motor (Fjortoft, 2001), la actitud hacia el medio ambiente (Asah, Bengston y Westphal, 2012) o la formación de los educadores (Schenetti y Guerra, 2018). No obstante, consideramos que no se ha prestado suficiente interés a cómo estos contextos contribuyen a la enseñanza-aprendizaje de las ciencias en las primeras etapas de escolarización cuando, a priori, parecen ser un laboratorio natural y vivo donde poder activar numerosas incógnitas, descubrir paisajes, especies vegetales y animales, instrumentos y materiales, con los que promover los primeros acercamientos al mundo de la ciencia.

En este trabajo presentamos una investigación realizada en la Scuola nel Bosco de Villa Ghigi en Bolonia (Italia), que aborda esta relación entre aprendizaje de las ciencias y espacios al aire libre.

\section{Ciencia y su aprendizaje en las primeras etapas de escolarización}

Tradicionalmente la Educación Infantil ha prestado más atención a las matemáticas o a la lectoescritura y no tanto a la enseñanza de las ciencias (Gómez-Motilla y Ruiz-Gallardo, 2016), lo que ha provocado una carencia de investigaciones en este campo (Cantó Doménech, de Pro Bueno, y Solbes, 2016).

No obstante, son varias las voces que apoyan la idea de incluir la educación científica desde los primeros años (Claxton, 1994; Cortés et al., 2012), pues es necesaria para el adecuado desarrollo de los niños (Trundle, 2010) y un componente esencial para una educación infantil de calidad (Brenneman, Stevenson-Boyd y Frede, 2009). Las edades tempranas son fundamentales para motivar el pensamiento científico, aprovechando la curiosidad e interés innato de los niños por el mundo que les rodea (Amaro, Manzanal y Cuetos, 2015) y su tendencia natural a disfrutar observando la naturaleza (Trundle, 2010). Características que deben tomarse en consideración para propiciar y promover estos primeros acercamientos a la ciencia, máxime con los datos que apuntan hacia la disminución gradual del interés por el estudio de la misma en etapas posteriores (COSCE, 2011).

$\mathrm{Al}$ contrario de lo que se pensaba, se ha constatado que los niños antes de llegar a la escuela poseen conocimientos sobre el medio natural (McClure et al., 2017) y cuentan con competencias científicas (Brenneman et al. 2009). Asimismo, el proceso por el cual elaboran respuestas a los diferentes interrogantes que genera su interacción con el mundo presenta semejanzas con el método científico, tal como demuestran investigaciones recientes (Duschl, Schweingruber y Shouse, 2007). De manera que su construcción del conocimiento se inicia como actividad de investigación (Greenfield, 2017). Así desarrollan teorías explicativas, se interrogan sobre fenómenos, formulan hipótesis y realizan inferencias (Gopnik, 2012). Son capaces de procesar de manera compleja sus experiencias y crear representaciones (French, 2004). Están biológicamente preparados para aprender del entorno en el que viven, sus experiencias en este son la base de su desarrollo (Canedo, Castellò y García Wherle, 2005).

En interacción con el ambiente, los niños elaboran conocimientos e interpretaciones sobre la vida cotidiana. Ambos supondrán los cimientos para la comprensión y formalización de 
conocimientos más complejos, muchos de ellos vinculados con las ciencias. El pensamiento científico los ayudará a resolver los interrogantes cotidianos que generan estas interacciones, por lo que es importante sembrar en ellos la idea de la importancia de la ciencia en su cotidianidad (Cruz-Guzmán, García-Carmona y Criado, 2017).

La enseñanza de las ciencias es la responsable de estimular este pensamiento científico empleando diferentes estrategias para potenciar la curiosidad, flexibilidad, persistencia, imaginación e inventiva de los niños (Brenneman et al. 2009).

A estas edades los pequeños toman conciencia de los fenómenos naturales, del cambio de las estaciones, conocen los animales y las partes de las plantas y reconocen los cambios esencialmente a través de los sentidos, por lo que los contenidos a trabajar deben ser perceptibles por el niño y atractivos (García-Carmona, Criado y Cañal, 2014). Estos contenidos deben estar más centrados en la creación de hábitos, procedimientos y actitudes positivas hacia el conocimiento de la ciencia y el entorno, que hacia una perspectiva conceptual. Ya que, a estos niveles madurativos, el conocimiento tiene un carácter eminentemente pragmático.

En este sentido se debe trabajar la observación, la formulación de preguntas, las predicciones, la planificación y desarrollo de investigaciones, el análisis, las habilidades matemáticas, la recolección e interpretación de datos, la construcción de explicaciones, la comunicación de la información, etc. (Greenfield, 2017). Sin olvidar la importancia de favorecer el sentimiento de pertenencia valoración, cuidado y respeto hacia el medio natural.

En resumen, los niños a estas etapas construyen sus propias interpretaciones del mundo, en gran medida, a partir de las experiencias sensoriales, el juego y la manipulación. Por ello, dónde y cómo desarrollen estas experiencias son cuestiones que condicionarán estas interpretaciones. Hecho del que se deriva la importancia de analizar cómo influye un determinado contexto en el desarrollo del pensamiento científico a estas etapas.

El bosque es un escenario donde pueden contextualizarse estos conocimientos y favorecer su aprendizaje. Las escuelas en espacios abiertos, cuyos horarios y programaciones suelen gozar de mayor flexibilidad, se presentan como un contexto que puede favorecer el acercamiento a las ciencias.

\section{Escuelas al aire libre en la etapa infantil}

El contacto con la naturaleza fortalece los lazos de los niños con su entorno (Torres-Porras, Alcántara, Arrebola, Rubio, y Mora, 2016). No obstante, la interacción con el medio físico y social, hasta hace poco asegurada, es hoy casi inexistente, pues los niños carecen de autonomía (Bertolino, Guerra, Schenetti y Antonietti, 2017). De manera que es necesario generar oportunidades para que estas interacciones tengan lugar.

La formación en la etapa infantil debe ofrecer un contexto rico para la experimentación que facilite oportunidades para su desarrollo y la resolución autónoma de situaciones complejas (Sánchez y González, 2016), pues entre los 4-6 años las experiencias que viven los pequeños son decisivas para su desarrollo social, cognitivo y operativo (Tonucci, 2001).

En este sentido, las escuelas en espacios naturales abiertos ofrecen multitud de estímulos. La naturaleza cambia constantemente y los niños son testigos de estos cambios, los observan y cuestionan, al contrario de lo que puede ocurrir en un aula convencional. Estas experiencias posibilitan la convivencia con diferentes especies de animales y plantas por las cuales, de forma natural, se sienten profundamente atraídos. Asimismo, proporcionan laboratorios donde desarrollar experimentos en contacto directo con los sujetos o elementos a investigar. 
Esta convivencia e interacción ayuda a los infantes a incorporar conocimientos científicos mediante experiencias directas (Freire, 2011). Por otro lado, el contacto con la naturaleza provoca placer a los pequeños, manifestándose este como una expresión de su deseo innato de conocer lo que les rodea (Eshach y Fried, 2005).

Como no existen dos aulas iguales, no hay dos escuelas al aire libre iguales. Los contextos son diferentes y también lo son educadores e infantes. No obstante, estos centros comparten características y líneas metodológicas que configuran su esencia. Una particularidad común es que carecen de instalaciones estándares, la mayoría solo cuenta con un edificio que sirve de apoyo para momentos de clima extremo y para almacenar ciertos materiales (Bruchner, 2012).

Estos centros se caracterizan por el constante equilibrio entre momentos poco o nada estructurados, donde se fomenta la autonomía de los pequeños, y momentos estructurados con intervención del educador (Bruchner, 2017).

La jornada se organiza en torno a unos momentos. El saludo matutino donde se organiza la jornada; los senderos donde los niños se desplazan a su propio ritmo; el juego libre en diferentes espacios y horarios; las actividades programadas (en algunos casos); el almuerzo y la despedida cuyo fin es reflexionar sobre lo acontecido. Esta estructuración en momentos transmite a los pequeños seguridad y confianza proporcionándoles certeza de lo que ocurrirá después, lo cual les permite concentrarse en la tarea que están desarrollando. Asimismo, existen unas reglas que los niños deben cumplir como el contacto visual con un adulto, seguir las reglas sociales, ser respetuosos con la naturaleza, no sobrepasar los límites del área establecida para el juego y acudir a la llamada de los adultos (Schenetti et al 2015).

En los diferentes momentos se desarrollan actividades que van desde la lectura, construcciones con materiales naturales, observación de fenómenos naturales, especies animales y vegetales, recolección de frutas de temporada o caminatas por el bosque. Estas actividades al aire libre conllevan efectos positivos sobre el desarrollo cognitivo de los niños (Wells, 2000), estimulan sus sentidos, la creatividad, el descubrimiento y la asunción de riesgos (Dadvand et al., 2018), lo cual coincide con características que contribuyen a la formación en la competencia científica.

\section{Problema y metodología}

El problema que orienta esta investigación se centra en conocer cómo contribuye la escuela en el bosque al aprendizaje de las ciencias en las primeras etapas de escolarización. Para abordar este problema se profundiza en torno a dos cuestiones:

- ¿Qué situaciones se generan en la escuela del bosque que promueven el aprendizaje de las ciencias?

- ¿Qué conocimientos de ciencias se ponen en juego en estos contextos al aire libre?

Para dar respuesta al problema formulado se diseñó una investigación de enfoque cualitativo. Esta permite estudiar fenómenos en su contexto natural, a los que se intenta dar sentido a partir del análisis de los datos (Denzin y Lincoln, 2005). Se empleó como estrategia un estudio de caso de naturaleza descriptiva (Álvarez y San Fabián, 2012), que no pretende generalizar los resultados.

El contexto de estudio fue la Scuola nel Bosco Villa Guigi (Bolonia, Italia) que se desarrolla desde 2011 en colaboración entre la Fundación del mismo nombre ${ }^{1}$, el Ayuntamiento y la Universidad de Bolonia, su objetivo principal es ofrecer un contacto directo entre niños y naturaleza. Esta escuela consta de dos tipos de actuaciones. Una se desarrolla durante el curso

\footnotetext{
${ }^{1}$ https://www.fondazionevillaghigi.it/
} 
escolar con una duración de uno o dos días. En ella participan niños, maestros y educadores de escuelas públicas en un proyecto conjunto con los educadores de la fundación. La otra es la escuela de verano, donde participan niños de forma voluntaria y educadores de esta fundación. En este caso los periodos son de 15 días.

Para el estudio, se seleccionó una de estas actuaciones. En concreto 15 días del mes de julio de 2017. En la experiencia participaron 26 niños $^{2} ; 2$ educadores ambientales con formación en escuelas al aire libre y un biólogo, maestro en formación ${ }^{3}$, todos pertenecen a la plantilla de la fundación. Las jornadas en el bosque se organizan en torno a diferentes momentos: el círculo, matinal y final, el juego libre y los senderos. En cada uno se generan situaciones con potencialidad para el aprendizaje de las ciencias, estas fueron el objeto del presente estudio.

La recogida de información se realizó a través de la observación participante de la investigadora que se integró en el proceso. Se emplearon diferentes fuentes para obtener una visión más rica y diversa de las situaciones:

- Guion de observación ${ }^{4}$. Este se adaptó a los objetivos de la investigación y fue validado. Sus ítems fueron: situación desencadenante, comportamiento de los niños, de los educadores y materiales empleados. Se complementó con el diario de investigación.

- Registro audiovisual. Posteriormente transcrito, que recoge situaciones significativas del proceso.

- Respuestas de los pequeños sobre las preguntas. Hechas por la investigadora y relativas a la experiencia vivida.

Tras la toma de datos, se procedió a la selección y reducción de los mismos bajo el criterio de las cuestiones de investigación, excluyendo y reservando aquellos datos que no estaban estrictamente relacionados con ellas. Los datos obtenidos se trataron desde el análisis de contenido. Para definir e identificar las unidades de análisis, se usó el procedimiento de unidades temáticas (Krippendorff, 1990).

Las unidades de información se organizaron en torno a las preguntas de investigación usando los momentos señalados para estructurarlas. En cada uno se seleccionaron y clasificaron las situaciones y conocimientos emergentes relacionadas con las ciencias, así como las situaciones promovidas por preguntas de niños y/o educadores ante hechos concretos que generaron situaciones de aprendizaje (por ejemplo, la aparición de una avispa).

Para el procesado de datos se empleó el software Nvivo9. Este permite obtener, entre otras cuestiones, las frecuencias de los diferentes elementos analizados.

En la tabla 1 se muestran los códigos empleados para el instrumento del cual se ha obtenido la información, así como el sujeto del que procede.

Tabla 1. Códigos instrumentos de recogida de información y sujetos

\begin{tabular}{|l|l|l|l|}
\hline Instrumentos & Sujetos \\
\hline $\begin{array}{l}\text { Guion observación y diario } \\
\text { investigación }\end{array}$ & GO & Educador 1 & EE \\
\hline Registro audiovisual & RA & Educador 2 & EA \\
\hline Preguntas a los niños & P & Educador 3 & EO \\
\hline & $\begin{array}{l}\text { Niño: Inicial } \\
\text { nombre+edad }\end{array}$ & F6 \\
\cline { 2 - 4 }
\end{tabular}

${ }^{2}$ De ellos: 1 niña de 2 años; 2 niños de 3; de 4 años: 7 niños y 7 niñas; de 5 años: 3 niños y 4 niñas; 2 niños de 6 años.

${ }^{3}$ Los participantes fueron debidamente informados y dieron su consentimiento para la toma de datos.

${ }^{4}$ El guion empleado es parte del proyecto de investigación "Nell'aula la scuola” desarrollo por la rd SIRQ y la Universidad de Turín. 


\section{Resultados}

Los resultados se muestran en torno a las cuestiones de investigación.

\section{Situaciones generadas en el contexto que promueven el aprendizaje de las ciencias}

Durante la investigación se identificaron tres momentos que organizan la jornada: el círculo, los senderos y el juego libre. En torno a ellos se desarrollaron un total de 74 situaciones significativas relacionadas con el aprendizaje de las ciencias. De ellas 16 pertenecen al círculo, 22 a los senderos, 28 al juego libre y 8 a otras situaciones que surgieron en el proceso y que pueden contribuir al aprendizaje de las ciencias. A continuación, se ilustran algunas de ellas.

\section{a) El círculo}

El círculo se emplea para iniciar el día y cerrarlo. Se desarrolla siempre en la misma zona del bosque.

La jornada comienza con el círculo matutino a la sombra de un álamo donde niños y educadores saludan al bosque con una canción. Después se comparten experiencias tanto del día anterior como ajenas al bosque. Además, se decide conjuntamente el programa del día.

Durante los círculos, se identificaron distintas situaciones que propiciaron el acercamiento al aprendizaje de las ciencias. Se exponen a continuación algunos ejemplos.

Uno de los días, la intervención de una educadora desencadena una situación significativa. Esta pregunta a los niños sobre los cambios que se observan de un día para otro en el espacio del círculo [RA].

- Veo que el sitio de reunión está un poco cambiado ¿no os parece? [EE]

- Está todo lleno de hojas y de ramas que ayer no estaban [T2]

- ¿Por qué están aquí? [EE]

- Porque ayer hubo tormenta y se han caído [CA]

- Porque el viento era fuerte [A5]

- Un rayo los ha golpeado [R4]

- Bravo habéis estado muy atentos" [EE]

Como se infiere de la secuencia, la educadora llama la atención sobre los cambios evidentes en el sitio de acogida y los pequeños relacionan estos cambios con los fenómenos atmosféricos acontecidos. Las preguntas son una herramienta que estimula a los pequeños en la construcción de conjeturas.

Esta situación generada en el círculo matutino propicia un diálogo entre los pequeños en torno al "desorden" generado por la tormenta. Una de las niñas [A5] propone hacer una recogida diferenciada entre hojas y ramas, aplaudida por educadores y niños. De manera que todos espontáneamente hacen esta clasificación.

A diferencia del círculo inicial, el final tiene como función reflexionar sobre lo acontecido durante la jornada, sobre las cosas descubiertas y aprendidas. Se emplea también para despedirse del bosque. En esta última línea se ha detectado su principal uso. Durante los días de la experiencia, solo el último se destina a expresar lo que ha supuesto la misma. Asimismo, se interroga a los niños sobre los aprendizajes adquiridos [P]:

"No se deben matar los animales, yo antes aplastaba los insectos" [T5]

"A las hormigas les gusta la naturaleza" [B5]

"He aprendido qué es una avispa, qué una mosca y qué una abeja" [L4]

"Encontrar insectos. Los insectos vienen a mí porque soy rubio y parezco un girasol” [R4] 
"He aprendido cómo son los caracoles, los gusanos, los ciempiés y las mariposas" [B4]

"El nombre de las plantas y por qué pasan algunas cosas" [F4]

Como puede verse en las respuestas, muchos de los pequeños reflexionan en torno a conocimientos que emergen de su interacción con el medio natural. De las mismas, puede extraerse como durante esta experiencia se fomentó la observación, el reconocimiento de especies, se establecieron relaciones causa efecto y se promovieron actitudes proambientales.

\section{b) Los senderos}

A lo largo de una jornada en la Scuola nel Bosco Villa Ghigi se desarrollan mínimo tres senderos diferentes. El primero tras el círculo matutino hacia el lugar para pasar la mañana, el segundo hacia el espacio para el almuerzo y el último hacia el sitio de despedida. Al ser los tres senderos diferentes las especies y paisajes son diversos y también las oportunidades de aprendizaje.

Al contrario de lo que pudiera parecer, los senderos ofrecen ocasiones para la exploración, la identificación, la recolección o el diálogo con y entre los pequeños. De hecho, se detectaron circunstancias aprovechadas por los educadores para suscitar interés en los niños y otras donde el interés fue espontáneo.

En esta línea, durante un paseo, un grupo de niños [F6, F5, P4] encuentra dos escarabajos muertos. Los cogen, observan los colores, cuentan las patas y se interrogan sobre la especie. $\mathrm{Al}$ no encontrar una respuesta, recurren a una educadora [EA]. Esta les propone usar la guía de insectos. Primero les explica qué aspectos deben tener en cuenta para la identificación y luego los buscan. Una vez localizados, la educadora lee la descripción y los niños escuchan.

En el diálogo entre niños y educadora surge un nuevo dilema en torno al nombre científico del escarabajo. Tiene un nombre "raro" comentan los niños y [EA] explica que el nombre está en latín y tiene dos partes como los suyos, nombre y apellido.

Esta secuencia muestra como el propio interés de los niños les lleva a cuestionarse e intentar resolver por ellos mismos el interrogante, siendo el educador un apoyo en el proceso. Se trata del séptimo día de la experiencia, los niños están habituados a la forma de trabajo y al contexto. Al iniciar la caminata parten con una actitud de búsqueda sobre cosas que les interesan o que pueden encontrar por el camino.

\section{c) El juego libre}

Los espacios dedicados al juego libre proporcionan mayor diversidad de experiencias, lo cual se debe fundamentalmente a dos factores el propio contexto y el tiempo dedicado. Se desarrollaron principalmente en dos marcos, el bosque y el prado, y durante la mañana y la tarde.

En este tiempo los niños se mueven autónomamente y deciden a qué jugar y con quién, en función de sus intereses. Entretanto los educadores permanecen cercanos e interviniendo lo menos posible.

El bosque tiene un arroyo y está lleno de restos de poda. El prado es abierto, aunque cuenta con árboles y una fuente.

En el bosque, se capta [RA] la siguiente secuencia en la que los niños realizan conjeturas en torno a la respiración de una hormiga que metieron en una botella:

- “Puede respirar la hormiga dentro de la botella? [A5]

- No puede, el aire no entra [E4]

- ¿Por qué no puede? Se está moviendo [A5] 
- Sí respira mira no está cerrada y puede entrar aire” [E4]

El diálogo establecido muestra la capacidad de argumentar de los niños. A estas edades se hacen preguntas y formulan conjeturas, para intentar dar respuesta a los interrogantes que encuentran, las cuales emergen inmediatamente después de la pregunta. La formulación de diversas conjeturas implica considerar diferentes soluciones cuando se encentra un problema. Asimismo, la elaboración de explicaciones conlleva ejercitar habilidades cognitivas.

Durante el juego libre algunos educadores organizan exploraciones. Su objetivo suele ser buscar huellas de animales e identificarlos, descubrir madrigueras, distintos tipos de plantas o animales, así como fomentar la curiosidad de los niños y su capacidad de observación. En una de ellas un grupo encuentra un coleóptero, con sumo cuidado lo cogen, discuten sobre el nombre y proponen varias opciones. Saben que es un insecto, pero no qué tipo y buscan a la educadora para identificar la especie. [EA] les pide que observen sus partes, su forma, el color y el tamaño. Los interroga sobre la función de la forma de la boca. Los niños exponen diversos razonamientos al respecto:

$$
\begin{aligned}
& \text { - "Para caminar [AG] } \\
& \text { - Para comer [F6] } \\
& \text { - Para excavar" [A4] }
\end{aligned}
$$

Finalmente hallan la especie, escarabajo rinoceronte hembra, tras descartar varias de las opciones propuestas. Estos descartes se basan en las comparativas entre las diferentes fotografías de la guía y los datos recogidos en la observación del escarabajo.

En esta secuencia identificamos una versión simplificada del razonamiento científico. Hay una pregunta inicial, se predice que se trata un insecto y se formulan posibles respuestas, se observan las características, se busca información, se manipula y se comparan y contrastan informaciones hasta obtener una conclusión plausible. La observación directa del insecto facilitó la identificación, generó una situación en la cual los niños debieron usar diferentes estrategias como conteo de patas, búsqueda en la guía, comparación de partes y formas y construcción de razonamientos respecto a la funcionalidad de las mismas.

Durante el juego libre, también se realizaron experimentos espontáneos en el prado. Por ejemplo, en una ocasión [EE] leía un libro a un grupo de niños y apareció una avispa que se posó en un resto de mortadela. Tras el alboroto inicial, se llama a la calma y se les pide que observen el comportamiento de la avispa. Uno de ellos comenta:

- "Está comiendo [M4]

- ¿Seguro? Fijaos bien [EE]

- No, solo está mordiendo ¿por qué? [B5]

- No tiene hambre [B3]

- ¿Y entonces para qué muerde? ¡Se va! [M4]

- Porque va a llevarle comida a sus larvas que son carnívoras. Hagamos un experimento ¿pensáis que volverá? [EE]

- Sí volverá porque queda carne [B5]

- No creo que sepa volver" [R4]

Los niños permanecen atentos y pasados unos minutos aparece de nuevo:

- "iHa vuelto! [R4]

- Se va de nuevo, veamos si vuelve" [EE]

Pasados unos momentos la avispa vuelve: 
- Está aquí, pero es otra, está más gorda [M4]

- ¡Es verdad! ¡Es otra!” [B4]

[EE] les explica que la primera avispa ha informado al resto del avispero sobre donde está la comida y por eso ha venido una distinta. Los invita a seguir observando y durante este tiempo llegan cuatro avispas más que repiten la misma operación que la primera.

En esta secuencia el grupo de niños formuló conjeturas sobre el comportamiento de la avispa, sobre la posibilidad de su retorno, practicó la espera y la observación, identificó diferencias entre individuos. Además, los comentarios de la educadora los acercó al conocimiento del comportamiento de las avispas.

\section{d) Otras situaciones significativas}

$\mathrm{Al}$ margen de los tres momentos estructurantes, se percibieron cuestiones de relevancia durante los almuerzos y meriendas.

Cabe destacar el uso responsable del agua, tanto para beber como en la higiene. Antes de comer los niños se organizan en filas para lavarse las manos en cubos y se emplea jabón natural. En relación al consumo, solo hay una fuente a la que los niños tienen acceso a la hora de la comida, el resto del tiempo deben gestionar el agua.

Asimismo, se utilizaron recursos naturales a modo de pinturas (sabuco) y hojas y ramas para crear adornos. Otro hecho destacable es el consumo de frutas y verduras de temporada.

\section{Conocimientos puestos en juego durante la experiencia}

Para contestar a esta cuestión se presenta una clasificación en torno a: conceptos, procedimientos y actitudes relacionados con la actividad científica y el medio natural, y otros conocimientos que pueden contribuir al aprendizaje de las ciencias. Esta clasificación permite reconocer que la enseñanza de las ciencias es más que trabajar sobre teorías o conceptos, pues pone de manifiesto la importancia de los procesos en la generación de conocimiento y de los valores asociados (Sanmartí, 2000).

$\mathrm{Si}$ bien es cierto que no es el principal objeto de este estudio analizar la significatividad de los contenidos, sino conocer si están presentes en el contexto de la escuela en el bosque, consideramos que las frecuencias detectadas sobre ellos, pueden apoyar esta línea. En total se identificaron 266 contenidos relacionados con ciencia, de tipo procedimental y conceptual, en el periodo analizado. La figura 1 muestra la frecuencia relativa de contenidos de ambos tipos, siendo los procedimentales los más significativos (64\% del total). Los de tipo actitudinal aparecen vinculados a todo el proceso y son difícilmente cuantificables, por tanto, se presentan de forma descriptiva. Por ejemplo: la curiosidad ante la aparición de cualquier cambio en el medio.

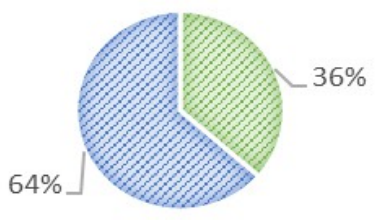

\footnotetext{
Conceptos relacionados con la actividad científica y el medio natural

- Procedimientos relacionados con la actividad científica y el medio natural
}

Figura 1.Frecuencia de los diferentes tipos de contenidos identificados.

Dentro de cada tipo se establecieron grupos, ejemplos de los conocimientos y la frecuencia del grupo en relación a los contenidos de cada tipo, en el caso de conceptos y procedimientos. 
Los de tipo conceptual se presentan en la tabla 2, clasificados en 7 grupos. Los más frecuentes son los relativos a nombres comunes, cuestión que emerge de la propia curiosidad de los niños por conocer aquello que les rodea. No sucede igual con los nombres científicos que son expuestos por los educadores. También destaca el uso de vocabulario científico, asociado a muchas de las actividades que se desarrollan en el bosque.

Tabla 2. Conceptos relacionados con la actividad científica y el medio natural

\begin{tabular}{|l|l|c|}
\hline Grupo & Ejemplos & Frecuencia \\
\hline $\begin{array}{l}\text { Nombres comunes de } \\
\text { animales, plantas y } \\
\text { frutos }\end{array}$ & $\begin{array}{l}\text { Corneja ceniza, abejorro, sírfido, álamo, cedro, tejo, tomate salvaje, } \\
\text { nueces, granadas }\end{array}$ & $24,7 \%$ \\
\hline $\begin{array}{l}\text { Nombres científicos de } \\
\text { animales }\end{array}$ & Lacanus cervus, Oryctes nasicornis & $2,2 \%$ \\
\hline $\begin{array}{l}\text { Seres inertes naturales } \\
\text { materiales naturales }\end{array}$ & $\begin{array}{l}\text { Rocas, minerales, agua, tronco, excremento, hoja, concha, } \\
\text { madrigueras }\end{array}$ & $16,5 \%$ \\
\hline Fenómenos naturales & Lluvia, tormenta, viento, nubes, rayo, relámpago, trueno & $14,4 \%$ \\
\hline Ciclo de la vida & Vivo, muerto, larvas, muda, respirar, comer & $16,5 \%$ \\
\hline Tipos de paisaje & Bosque, prado, montaña, ciudad & $8,2 \%$ \\
\hline Vocabulario científico & Experimento, hipótesis, datos, observación, análisis & $17,5 \%$ \\
\hline
\end{tabular}

En relación a los contenidos de carácter procedimental (tabla 3), estos son mayoritarios y sus grupos más ricos. Sobresalen las observaciones, la identificación, el reconocimiento de olores y las recolecciones. Todos ligados a hábitos que los niños adquirían conforme pasaban sus días en el bosque.

En lo que se refiere a los contenidos de naturaleza actitudinal se detectaron la curiosidad ante aquello que los rodea; la creatividad, por ejemplo, cuando se disponen a construir cabañas; la cooperación necesaria para ello; el respeto hacia los seres vivos e inertes; pero también hacia las opiniones de los demás; o a las reglas de comportamiento. Asimismo, en este tipo están aquellos relacionados con actitudes proambientales como el ahorro de agua; la reducción de residuos; el consumo de productos de temporada; o el uso de materiales naturales para dibujar, que tienen una presencia constante.

Por último, se identificaron contenidos más generales que pueden contribuir al aprendizaje de las ciencias: la toma de decisiones sobre el programa a seguir en la jornada, sobre las reglas a seguir en un juego; exposición de ideas o escuchaba activa. Es destacable también la adquisición de hábitos saludables como lavarse las manos antes de comer o limpiar una fruta recolectada antes de comerla.

A modo de resumen, los resultados reflejan pluralidad y abundancia de contenidos de ciencia presentes en el contexto de estudio. Sobresalen los de tipo procedimental sobre los conceptuales, tanto en cantidad como diversidad, lo cual tiene sentido puesto que a estas edades el aprendizaje es fundamentalmente de naturaleza práctica. Asimismo, ambos están complementados con el resto de contenidos que favorecen un aprendizaje más integral y contribuyen a promover la competencia científica. 
Tabla 3. Procedimientos relacionados con la actividad científica y el medio natural

\begin{tabular}{|c|c|c|}
\hline Grupo & Ejemplos & Frecuencia \\
\hline Observaciones & $\begin{array}{l}\text { Como parte de experimentos } \\
\text { Características de las especies } \\
\text { Alimentación de animales } \\
\text { Cambios atmosféricos }\end{array}$ & $10,7 \%$ \\
\hline Experimentos & $\begin{array}{l}\text { Experimento avispa y mortadela } \\
\text { Respiración de hormiga }\end{array}$ & $3 \%$ \\
\hline Comparaciones & $\begin{array}{l}\text { Insectos reales y guías } \\
\text { Ciruelas salvajes y ciruelas domésticas }\end{array}$ & $4,1 \%$ \\
\hline Clasificaciones & $\begin{array}{l}\text { Clasificación de hojas por tamaños } \\
\text { Clasificación diferenciada entre hojas y palos }\end{array}$ & $1,6 \%$ \\
\hline Toma de datos & $\begin{array}{l}\text { Identificación de coleópteros } \\
\text { Predicciones }\end{array}$ & $6,5 \%$ \\
\hline $\begin{array}{l}\text { Formulación de } \\
\text { conjeturas }\end{array}$ & A partir chicharra muerta se especula sobre su vuelo o no & $3,6 \%$ \\
\hline $\begin{array}{l}\text { Interpretación de } \\
\text { datos y elaboración } \\
\text { de conclusiones }\end{array}$ & $\begin{array}{l}\text { Respiración de hormiga } \\
\text { Tras la observación de las características morfológicas de un animal o } \\
\text { una planta }\end{array}$ & $8,3 \%$ \\
\hline Identificación & $\begin{array}{l}\text { Árboles y plantas a través de las hojas, tronco, tallo } \\
\text { Pájaros a través del canto } \\
\text { Nidos (insectos o pájaros) }\end{array}$ & $10,7 \%$ \\
\hline Relación & $\begin{array}{l}\text { Causa-efecto: huella con paso de un animal } \\
\text { Plumas con especie }\end{array}$ & $5,3 \%$ \\
\hline Reflexiones & Sobre lo aprendido, sobre ideas de los compañeros y educadores & $4,7 \%$ \\
\hline $\begin{array}{l}\text { Uso de recursos } \\
\text { científicos }\end{array}$ & Uso de guías para identificar especies & $5,3 \%$ \\
\hline Argumentación & $\begin{array}{l}\text { Respiración hormiga: dar explicaciones razonadas al fenómeno que } \\
\text { están observando } \\
\text { Construcción de un hormiguero: discusión sobre la forma más } \\
\text { adecuada }\end{array}$ & $5,3 \%$ \\
\hline $\begin{array}{l}\text { Resolución de } \\
\text { problemas }\end{array}$ & Búsqueda de herramientas para abrir frutos secos & $3,4 \%$ \\
\hline Predicciones & Ante fenómenos atmosféricos & $3 \%$ \\
\hline $\begin{array}{l}\text { Reconocimiento de } \\
\text { olores }\end{array}$ & Plantas, flores, tierra mojada & $10,7 \%$ \\
\hline Exploraciones & Incursiones autónomas o dirigidas para inquirir un lugar & $4,1 \%$ \\
\hline Recolecciones & $\begin{array}{l}\text { Frutos } \\
\text { Plantas medicinales } \\
\text { Materiales naturales }\end{array}$ & $10,7 \%$ \\
\hline
\end{tabular}

\section{Síntesis de resultados y conclusiones}

Los resultados obtenidos ponen de manifiesto que la Scuola nel Bosco Villa Ghigi es un contexto que favorece el acercamiento al aprendizaje de las ciencias, aún sin ser esta su principal finalidad.

En relación a las situaciones promotoras del aprendizaje de las ciencias, se identificaron diferentes hechos, espontáneos o generados por los educadores, donde los niños desplegaron habilidades científicas para dar soluciones a distintas problemáticas. Se observó cómo los pequeños generaron interrogantes, formularon conjeturas, razonaron, tomaron datos y los interpretaron, emplearon material científico, elaboraron posibles respuestas y llegaron a conclusiones.

$\mathrm{Si}$ bien es cierto que no son contenidos ajenos a otros contextos, este trabajo refleja que el bosque es un escenario que incita estos conocimientos y estimula el aprendizaje, potenciando e incidiendo en capacidades que los niños poseen innatamente como la curiosidad o la 
creatividad. Asimismo, una diferencia fundamental con otros marcos educativos tradicionales radica en que, a diferencia de estos donde las actividades científicas suelen ser programadas, en este caso emergen fundamentalmente de los niños fomentadas por la riqueza del propio bosque y la necesidad de satisfacer su curiosidad.

En un segundo plano, se encontraron diferencias en cuanto a la cantidad de situaciones generadas en los tres momentos estructurantes. En los senderos y el juego libre, se detectaron más situaciones relacionadas con el aprendizaje de las ciencias, en consecuencia, donde los conocimientos fueron más ricos. Los primeros fundamentalmente porque cambiaban, lo cual provocaba expectación en los infantes y los segundos porque ocupaban más tiempo y ofrecían más diversidad. Durante el juego libre, los niños tomaron decisiones sin la supervisión directa del adulto, desarrollaron diferentes iniciativas gracias a la autonomía que gozaban y pasaron más tiempo interactuando entre iguales. Además, la heterogeneidad de edades ayudó a la confrontación entre saberes.

Por otro lado, durante el juego libre hubo más tiempo para la intervención de los educadores que abundaron y profundizaron en cuestiones relacionadas con la ciencia. Si bien es cierto que los niños construyeron y resolvieron por sí mismos distintas problemáticas, quedó patente la necesidad de acompañamiento en el proceso.

Con respecto al círculo, el matutino se empleó para organizar la jornada y recordar algunas experiencias, pero su incidencia en la generación de situaciones para el aprendizaje de las ciencias fue menor. Por otro lado, el de despedida no cumplió con su función principal, afianzar, recordar y reflexionar sobre los acontecimientos del día. Lo cual no contribuye al proceso de aprendizaje puesto que la recapitulación es un paso necesario para la interiorización del conocimiento.

$\mathrm{Si}$ atendemos a los conocimientos puestos en juego, los resultados muestran un amplio abanico que desde una perspectiva globalizada los niños van interiorizando. Son los de tipo procedimental los de mayor protagonismo, seguidos de conceptuales y apoyados por los actitudinales, lo cual resulta lógico dado el carácter práctico del aprendizaje en estas etapas. Asimismo, destaca que se trata de contenidos apreciables claramente en el contexto y que atraen a los niños. No obstante, como decíamos líneas arriba, se detectó una carencia de procesos reflexivos que apoyen el fortalecimiento de los mismos, pues las reflexiones reflejadas fueron más de corte espontáneo y no promovidas por los educadores.

En otro orden de cosas, el estudio presenta una serie de limitaciones inherentes a su naturaleza. Se trata de una experiencia concreta en un contexto muy específico y por tanto no generalizable, asimismo el proceso conlleva diversidad de situaciones en paralelo que impidieron su seguimiento total. No obstante, ha abierto potenciales líneas de investigación: el análisis de la incidencia de la formación de los educadores en el proceso desarrollado, la necesidad de conocer porqué el circulo final no cumple con su misión reflexiva y la importancia de analizar la evolución en el aprendizaje. Por último, el estudio anima a analizar el estado de la cuestión en nuestro país, pues son escasas las investigaciones en este campo. Un ejemplo es el estudio realizado por Sanz Alonso et al (2018) que presenta un modelo de evaluación centrado en aspectos como la idoneidad de los espacios, el juego, las relaciones, el estrés, o la mejora de actitudes y conocimiento sobre el medio natural, cuestiones en consonancia con lo estudiado en esta investigación. Igualmente, existe una carencia de fuentes fiables para obtener datos relacionados con aspectos tales como homologación, etapas a las que se dirigen, formación del profesorado, distribución, entre otras cuestiones, que servirían para caracterizar este tipo de escuelas en el ámbito español. 
A modo de cierre, sin duda la escuela en el bosque fomenta la observación y el estudio de fenómenos naturales que no suelen estar presentes en el aula y lo hace desde situaciones reales. Favorece una aproximación a la ciencia donde los niños asimilan naturalmente la utilidad de la misma para dar respuesta a sus interrogantes, promueve la educación científica y estimula el pensamiento científico en edades tempranas.

Dado que los niños aprenden a partir de las propias experiencias, parece adecuado señalar que es necesario generar estas oportunidades en contextos ricos en estímulos como el bosque. No obstante, consideramos que estas experiencias educativas deben acompañarse de un programa de actividades que ayuden a sacar un mayor partido. En este sentido sería adecuado generar sinergias entre las clases de centros educativos convencionales y escuelas en espacios abiertos. La integración del entorno en la escuela es una idea defendida desde multitud de ámbitos. La experiencia analizada permite la interacción directa del niño con su medio cercano, lo significativo de este tipo de actividad y su necesaria integración en la práctica educativa. Por ello, optamos por la divulgación de actividades de esta naturaleza que permitan valorar a los maestros su potencialidad.

\section{Referencias}

Álvarez C., San Fabián J. L. (2012) 'La elección del estudio de caso en investigación educativa', Gazeta de Antropologia, 28(1), 1-12.

Amaro F., Manzanal A. I., Cuetos M. J. (2015) Didáctica de las Ciencias Naturales y Educación Ambiental en Educación Infantil. Logroño: unireditorial.

Asah S. T., Bengston D. N., Westphal, L. M. (2012) 'The Influence of Childhood: Operational Pathways to Adulthood Participation in Nature-Based Activities', Environment and Behavior, 44(4), 545-569.

Bertolino F., Guerra, M., Schenetti M., Antonietti M. (2017) 'Educazione e natura: radici profonde, sfide presenti, prospettive future'. En A. Bondioli, D. Savio, (Ed). Crescere bambini. Immagini d'infanzia in educazione e formazione degli adulti (pp. 61-77). Parma: Junior.

Brenneman K., Stevenson-Boyd J., Frede E. C. (2009) Early Mathematics and Science: Preschool Policy and Practice (Preschool Policy Brief No. 19). New Brunswick: National Institute for Early Education Research.

Bruchner, P. (2012) 'Escuelas infantiles al aire libre’, 26 Cuadernos de Pedagogía, 420, 1-4.

Bruchner, P. (2017) Bosquescuela. Guía para la educación infantil al aire libre. Cullera: Ediciones Rodeno.

Canedo S., Castellò J., García Wherle P. (2005) 'La construcción de significados científicos en la etapa de educación infantil: una experiencia con planos inclinados', Enseñanza de las Ciencias, $\mathrm{N}^{\mathrm{o}}$ Extra, 1-6.

Cantó Doménech J., de Pro Bueno A., Solbes, J. (2016) ¿¿Qué ciencias se enseñan y cómo se hace en las aulas de educación infantil? La visión de los maestros en formación inicial', Enseñanza de las Ciencias, 34(3), 25-50.

Claxton, G. (1994) Educar mentes curiosas. Madrid: Visor.

Cortés A. L., Gandara M., Calvo J. M., Martínez M. B., Gil M. J., Ibarra J., Adegui J. (2012) 'Expectativas, necesidades y oportunidades de los maestros en formación ante la enseñanza de las ciencias en la educación primaria', Enseñanza de las Ciencias, 30(3), 155176. 
COSCE (2011) Informe Enciende. Enseñanza de las Ciencias en la Didáctica Escolar para edades tempranas en España. Madrid: Confederación de Sociedades Científicas de España.

Cruz-Guzmán, M., García-Carmona, A., Criado A. M. (2017) 'Aprendiendo sobre los cambios de estado en educación infantil mediante secuencias de pregunta-prediccióncomprobación experimental', Enseñanza de las Ciencias, 35(3), 175-193.

Dadvand P., Pujol J., Macià D., Martínez-Vilavella G., Blanco-Hinojo L., Mortamais M., Sunyer J. (2018) 'The Association between Lifelong Greenspace Exposure and 3Dimensional Brain Magnetic Resonance Imaging in Barcelona Schoolchildren', Environmental Health Perspectives, 126(2), 1-8.

Denzin N. K., Lincoln Y. S. (2005) The Sage Handbook of Qualitative Research. (3 ${ }^{\mathrm{a}}$ Ed). Londres: Sage.

Duschl R. A., Schweingruber H. A., Shouse A. W. (2007) Taking science to school: learning and teaching science in grades K-8. Washington: National Academies Press.

Eshach H., Fried M. N. (2005) 'Should science be taught in early childhood?', Journal of Science Education and Technology, 14(3), 315-336.

Fjortoft I. (2001) 'The Natural Environment as a Playground for Children: The Impact of Outdoor Play Activities in Pre-Primary School Children.', Early Childhood Education Journal, 29(2), 111-117.

Freire H. (2011) Educar en verde. Ideas para acercar a los niños y niñas a la naturaleza. Barcelona: Graó.

French L. (2004) 'Science as the center of a coherent, integrated early childhood curriculum', Early Childhood Research Quarterly, 19, 138-149.

García-Carmona A., Criado A. M., Cañal, P. (2014) 'Alfabetización científica en la etapa 3-6 años: Un análisis de la regulación estatal de enseñanzas mínimas', Enseñanza de las Ciencias, 32(2), 131-149.

Gómez-Motilla C., Ruiz-Gallardo J.R. (2016) 'El rincón de la ciencia y la actitud hacia las ciencias en educación infantil', Revista Eureka sobre Enseñanza y Divulgación de las Ciencias, 13(3),643-666.

http://dx.doi.org/10.25267/Rev_Eureka_ensen_divulg_cienc.2016.v13.i3.10

Gopnik A. (2012) 'Scientific thinking in young children: Theoretical advances, empirical research, and policy implications', Science, 337(6102), 1623-1627.

Greenfield D. B. (2017) 'Unleashing the Power of Science in Early Childhood: A Foundation for High-Quality Interactions y Learning', Zero to Three, 37(5), 13-21.

Krippendorff K. (1990) Metodología de análisis de contenido. Barcelona: Paidós.

McClure E. R., Guernsey L., Clements D. H., Bales S. N., Nichols J., Kendall-Taylor N., Levine, M. H. (2017) STEM Starts Early: Grounding Science, Technology, Engineering, and Math Education in Early Childhood. New York: Joan Ganz Cooney Center at Sesame Workshop.

Sánchez S., González C. (2016) 'La asamblea en educación infantil: un espacio para crecer como grupo', Revista Iberoameriana de Educación, 71, 133-150.

Sanz Alonso J., Sistiaga M., Urkidi P., Andoño I., Orbegozo K. 2018. Educar en la naturaleza en infantil: modelo de evaluación para un bosque escuela. En Martínez Losada, C. y 
García Barros, S. (coord.). 28 Encuentros de Didáctica de las Ciencias Experimentales. Iluminando el cambio educativo (pp. 327-331). A Coruña: Universidade da Coruña.

Sanmartí, N. (2000). El diseño de unidades diácticas. En J. Perales y P. Cañal (Ed), Didáctica de las ciencias experimentales (pp. 239-266). Alcoy: Marfil.

Schenetti M., Guerra E. (2018) 'Emotion Map Making. Discovering Teachers' Relationships with Nature', Asia-Pacific Journal of Research in Early Childhood Education, 12(2), 31-56.

Schenetti M., Salvaterra I., Rossini B. (2015) La scuola nel bosco. Pedagogia, didattica e natura. Trento: Erickson.

Taylor A., Kuo F. E. (2010) 'Is contact with nature important for healthy child development? State of the evidence', in Spencer, C. and Blades, E. (eds) Children and Their evironments. Cambridge, UK: Cambridge University Press, 124-140.

Tonucci F. (2001) ‘CCómo introducir la investigación escolar?’, Investigación en la escuela, 43, 3950 .

Torres-Porras J., Alcántara J., Arrebola J. C., Rubio S. J., Mora M. (2016) 'Trabajando el acercamiento a la naturaleza de los niños y niñas en el Grado de Educación Infantil. Crucial en la sociedad actual', Revista Eureka sobre Enseñanza y Divulgación de las Ciencias, 14(1),258-270. http://dx.doi.org/10.25267/Rev_Eureka_ensen_divulg_cienc.2017.v14.i1.19

Trundle K. C (2010) 'Teaching science during the early chilhood years', in Best practices and research base.

Wells N. M. (2000) 'Effects of Greenness on Children's Cognitive Functioning', Environment and Behavior, 32(6), 775-795. 